\title{
Direct in-situ detection of sulfur loading on fixed bed catalysts
}

\author{
Peter Fremerey ${ }^{1,2}$, Andreas Jess ${ }^{1}$, Ralf Moos ${ }^{2}$ \\ ${ }^{1}$ Department of Chemical Engineering, University of Bayreuth, 95440 Bayreuth, Universitätsstraße 30, \\ Germany \\ Peter.Fremerey@uni-bayreuth.de \\ ${ }^{2}$ Department of Functional Materials, University of Bayreuth, 95440 Bayreuth, Universitätsstraße 30, \\ Germany
}

\begin{abstract}
:
The aim of this work is to develop a sensor concept that detects in-situ the sulfur poisoning level of a fixed bed catalyst during deactivation by sulfur compounds. Therefore, single catalyst pellets are contacted and their electrical impedance is measured directly in the reactor. Since sensor pellets and catalyst particles in the fixed bed are identical, it can be assumed that the sulfur affects the sensor and the catalyst particles the same way at equal test conditions. The impedance of the sensors in the unloaded state is in the range of some $\mathrm{M} \Omega$ and decreases continuously by decades as soon as hydrogen sulfide is added to the gas stream. Using a product gas analysis, the sorbed sulfur mass can be calculated through a mass balance. In order to verify the data, also weighing experiments are conducted with the fixed bed. In addition to these techniques, XRD and EDX measurement are used to analyze the exact way of sulfur poisoning.
\end{abstract}

Key words: in-situ detection, sulfur poisoning, hydrogen sulfide, single catalyst pellets, fixed bed catalyst, electrical impedance

\section{Introduction}

Heterogeneous catalysts are used to enhance the rate of chemical reactions and to increase the selectivity to desired products. In many cases, catalysts deactivate with time on stream, which may be caused by the reaction of active compounds of the catalyst with components of the gas stream. For example, metal catalysts may be poisoned by sulfur compounds such as hydrogen sulfide $\left(\mathrm{H}_{2} \mathrm{~S}\right)$ either by chemisorption on the catalyst surface or by the formation of bulk sulfides such as $\mathrm{Ni}_{3} \mathrm{~S}_{2}$ and FeS [1]. Sulfur poisoning occurs during reformation of heavy gasoline, during methanation based on synthesis gas from coal gasification, and in many other refinery and petrochemical processes [2, 3].

Often, no actual information about the catalyst state exists, and deactivation can only be determined indirectly from the change of the reaction rate or degree of conversion. To optimize the processes, the knowledge of the actual sulfur loading can be helpful. Therefore, a method to detect in-situ the sulfur poisoning level has been investigated in this work.

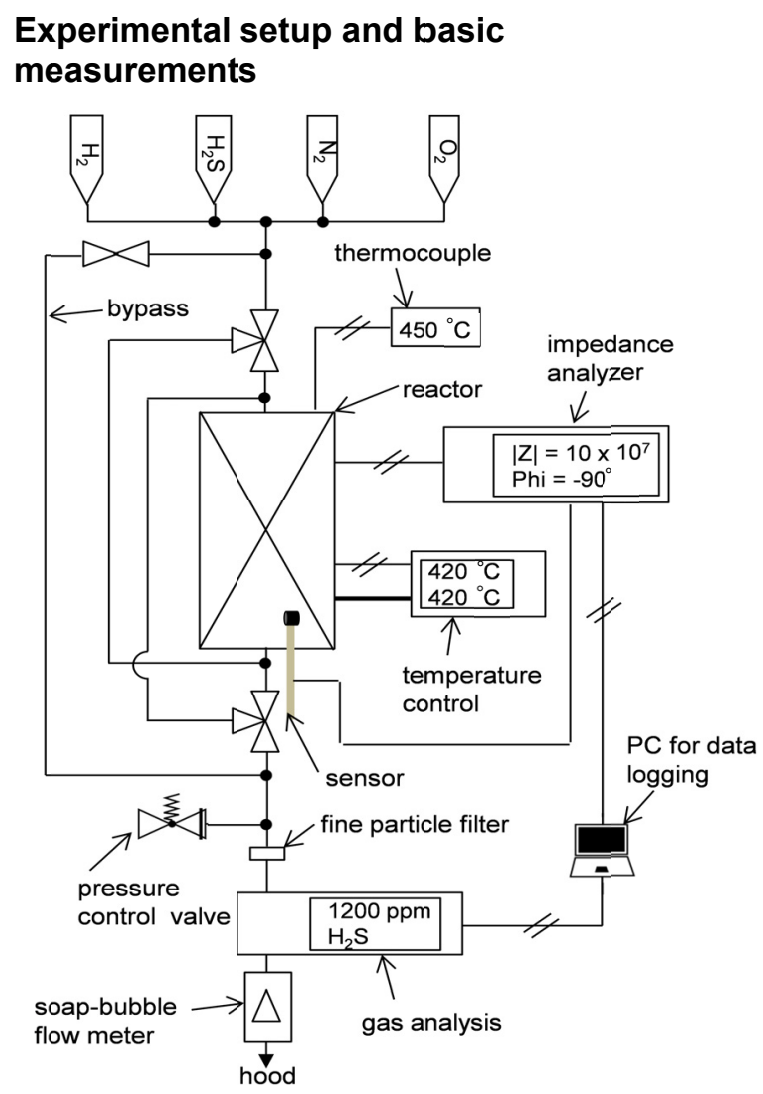

Fig. 1: Flow diagram of the experimental setup 
The tests are accomplished in a quartz glass reactor. Figure 1 shows schematically the experimental set-up. The reactor consists of a hollow glass cylinder with a glass frit in the middle of the tube to place a fixed bed on it. The sensor is placed in the fixed bed near the reactor inlet. The reactor is heated to an operation temperature of $450{ }^{\circ} \mathrm{C}$ at a gas flow rate of $\dot{V}_{\text {total }}=50 \mathrm{l} / \mathrm{h}$ at atmospheric pressure. According to Iemperature Programmed Desorption measurements (ChemBet 3000, Quantachrome Instruments), the effect of sintering for dispersed nickel atoms is negligible at this temperature. Because of such undesirable effects through the temperature it is important that the sensor and the fixed bed have the same temperature. For sulfurization tests, a concentration of $\mathrm{H}_{2} \mathrm{~S}$ of approximately $1200 \mathrm{ppm}$ in $\mathrm{N}_{2}$ was chosen. Such a high concentration enables the formation of nickel sulfide since chemisorption only takes place at very low concentrations of a few ppm of $\mathrm{H}_{2} \mathrm{~S}$ [1].

The basic idea to use a catalyst particle sensor is that the sensing material changes its structure the same way as the catalyst particles in the fixed bed do. The sensor setup consisting of catalyst pellets is already shown for conductometric coke loading sensors $[4,5]$. The catalyst used here consists of 37 wt-percent $\mathrm{Ni}$ on $\mathrm{SiO}_{2}$ [6]. To observe the electrical properties of the sensor particle, the flat planes of the sensor particle are coated with gold paste (DuPont KD 5744L) and are contacted with gold wires fed through the reactor by a ceramic capillary tube (Friatec). For stability, the sensor particle is screwed in a steel case (Fig. 2). All drills are sealed with ceramic glue (Resbond 919; Polytec) in order to get the setup gastight.

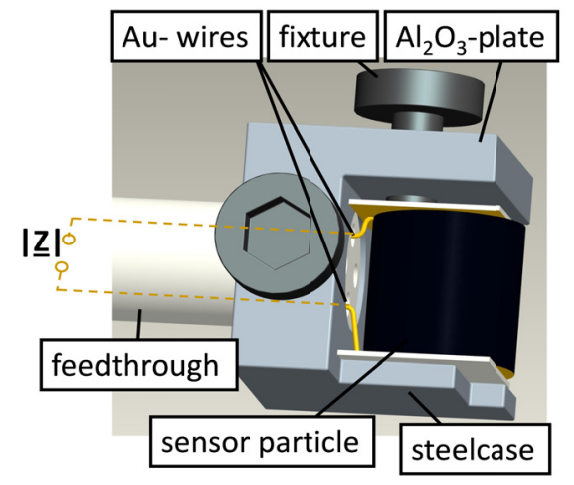

Fig. 2: CAD-drawing of the catalyst particle sensor

The actual state of the catalytic fixed bed during the deactivation by $\mathrm{H}_{2} \mathrm{~S}$ is measured by the electrical impedance at a single catalyst pellet. The electrical conductances of the sensor particles change with sulfur poisoning, as can be seen by the complex impedance $Z$ (Fig. 3). At first, $Z$ is measured in a range between $1 \mathrm{~Hz}$ and $10 \mathrm{MHz}$ to characterize the whole system in the different sulfurized states.

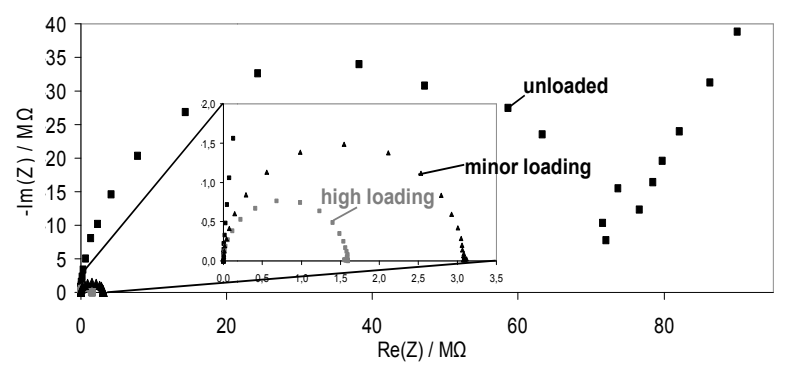

Fig. 3: Impedance of a sensor with different amounts of sulfur loading at $450{ }^{\circ} \mathrm{C}$

The measured impedance spectrum of a sensor particle reveals a semicircle in the complex plane. This can be modeled by an equivalent parallel circuit model consisting of a conductance, $G$, and a capacitor. The impedance decreases with sulfur poisoning which is indicated through the reduced diameter of the semi circle in Fig. 3. For the evaluation of the stored sulfur mass, a gas analysis (NGA 2000 MLT 3; Emerson) is used. The sulfur mass balance is calculated from:

$$
m_{S}=\frac{\dot{V}_{\text {total }} \int_{0}^{t_{\text {end }}}\left(y_{\mathrm{H}_{2} S, \text { in }}-y_{\mathrm{H}_{2} S, \text { out }}\right) \mathrm{d} t}{V_{m}} M_{s}
$$

$V_{\mathrm{m}}$ is the molar gas volume, $y_{\mathrm{H} 2 \mathrm{~S}}$ the molar fraction, and $M_{\mathrm{s}}$ the molar mass of sulfur.

\section{First measurement results}

Fig. 4 shows the sulfur poisoning process of a fixed bed nickel catalyst at $1 \mathrm{kHz}$. The conductance, $G$, of the sensor is displayed on the left $y$-axis and the sulfur mass, $m_{\mathrm{S}}$, that is stored in the fixed bed and in the sensor particle on the right axis.

During sulfur loading, the conductance increases strongly during the first $30 \mathrm{~s}$, which reflects the change from capacitive to resistive behavior occurs. Then, the slope of the conductance decreases and the behavior is almost linear for $5 \mathrm{~h}$.

Even though nickel particles are dispersed at the external surface and within the pores of the catalyst particles, the conductance of the pellets prior to sulfur exposure in the non-poisoned state is very low (Fig. 3). It is assumed that this is due to the high dispersion of the nickel in the catalyst resulting, i.e., no percolation (conductive paths) between the gold contacts exist. The formation of nickel sulfides is expected to enhance the volume of the dispersed nickel particles and therefore to decrease the distance between the conductive 


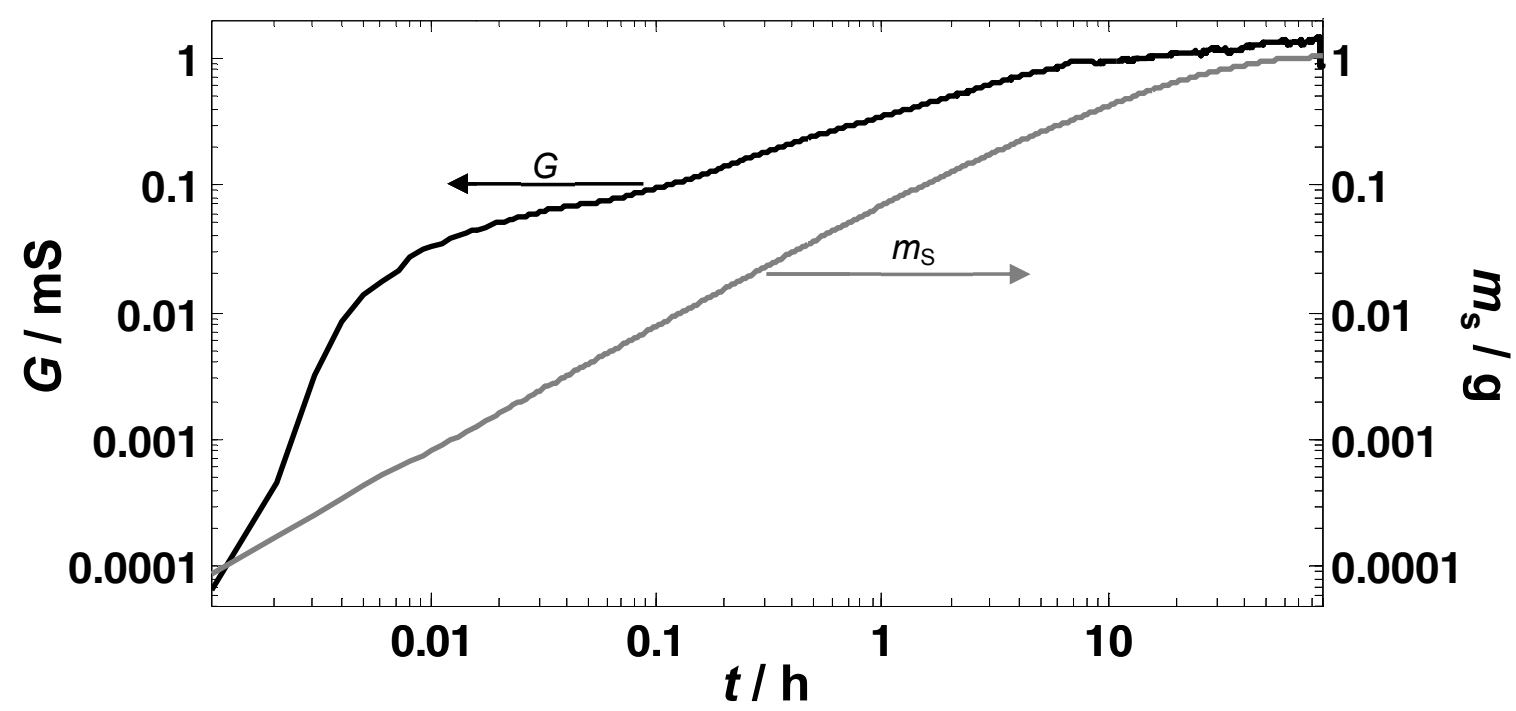

Fig. 4: Sulfur loading experiment: conductance, G (black), of the sensor catalyst particle and stored sulfur mass, $m_{S}$ (grey), in the fixed bed calculated acc. to equation 1

sites. It is known from [7], that the molar volume of nickel is $6.6 \mathrm{~cm}^{3} / \mathrm{mol}$ compared to nickel sulfide with a value of $17.3 \mathrm{~cm}^{3} / \mathrm{mol}$ [8]. Hence, the immediate increase in the conductance upon sulfur exposure can be attributed to this volume change resulting in the formation of the conductive paths. The applied $\mathrm{H}_{2} \mathrm{~S}$ concentration seems to be high enough to form rapidly electrical connections on the catalyst surface resulting in a resistive behavior. The following almost linear increase in the conductance might be caused by the diffusion of the sulfur into the pellet to accessible nickel sites until saturation occurs.

In this application, the curve of the stored mass of sulfur in the fixed bed shows a nearly linear slope up to at least $1 \mathrm{~h}$ of sulfur exposure. Thereafter, the slope of the curve decreases very slowly, which is an indication of a diffusionlimited reaction. Probably the first linear slope is connected with the catalyst filling in the reactor that is loaded with sulfur from the bottom to the top of the fixed bed. The calculation of the final sulfur load of the catalyst based on the data of the gas analysis leads to a molar Ni-to-S ratio of 1:1. X-ray diffraction (XRD) analysis supports this statement, where in addition to NiS only $\mathrm{Si}_{2} \mathrm{O}_{3}$ and a small amount of elementary nickel could be detected.

Energy dispersive X-ray (EDX) analyses also show that only NiS is formed for the given experimental conditions with a relative high concentration of $\mathrm{H}_{2} \mathrm{~S}$, and that a definite sulfur front moves from the external surface towards the center of the particle. In the area where nickel sulfide is found, the amount of sulfur is almost the same as the amount of nickel. In figure 5 , a probe is displayed where the $\mathrm{H}_{2} \mathrm{~S}$ concentration was around $1200 \mathrm{ppm}$. In contrast to the previous measurement, the sulfur poisoning time in Fig. 5 was much shorter. The diagram shows the counts of sulfur detected by the EDX analysis in a line scan along a catalyst particle.

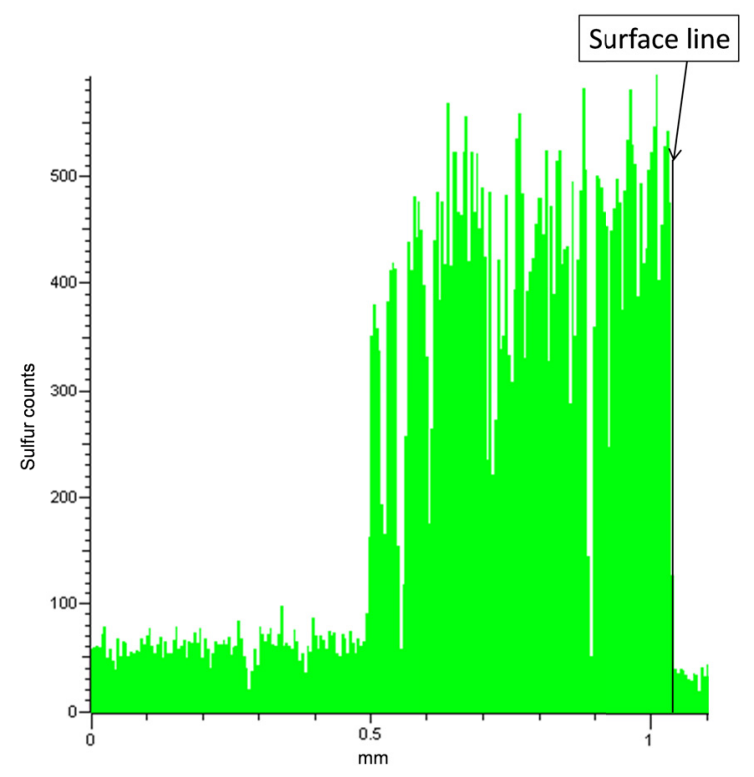

Fig. 5: Sulfur counts over a line from the catalyst particle inside up to the surface

The black line at $1.04 \mathrm{~mm}$ marks the surface of the cylindrical pellet. The data zero designates a point inside the particle on the line to the particle center. The data shows a nearly definite detected sulfur front. Fix point measurements with EDX along this line allow to calculate atom percent. The amounts of nickel and sulfur at these points equates approximately to the quotient of NiS which is in accordance to XRD analysis. A disadvantage of this technique is 
that a measurement gives only an almost exact result for one point. Therefore, more experiments must be conducted to approve these data.

\section{Conclusion and outlook}

It was shown that the investigated sensor system is able to detect in-situ the sulfur poisoning of a nickel catalyst with $\mathrm{SiO}_{2}$ as supporting material. The impedance decreases with a continuous formation of nickel sulfides. A detailed correlation between the sulfur loading and the electrical signal is under investigation. Therefore the conductance of pellets consisting of nickel, silicon oxide, nickel oxide and different nickel sulfides is measured to compare these results with the measurement from the catalyst material. Furthermore, it is important to investigate the dependence of the sensor signal on varying test parameters (e.g. lower sulfur concentration, $\mathrm{SO}_{2}$ instead of $\mathrm{H}_{2} \mathrm{~S}$ for poisoning, temperature) to understand how the sulfur poisoning proceed. Also the regeneration of the catalyst pellets should be analyzed.

\section{Acknowledgements}

The authors A.J. and R.M. are indebted to the German Research Foundation (DFG) for financial support (Je 257/12-2, Mo 1060/5-2) respectively.

\section{References}

[1] J.R. Rostrup-Nielsen, Chemisorption of hydrogen sulfide on a supported nickel catalyst, Journal of Catalysis 11, 220-227 (1986); doi: 10.1016/00219517(68)90035-3

[2] J. Oudar, Sulfur Adsorption and Poisoning of Metallic Catalysts, Catalysis Reviews 22, 171195 (1980); doi: 10.1080/03602458008066533

[3] C.H. Bartholomew, G.D. Weatherbee, G.A. Jarvi, Sulfur poisoning of nickel methanation catalysts, Journal of Catalysis 60, 257-269, 1979; doi: 10.1016/0021-9517(79)90147-7

[4] N. Müller, C. Kern, R. Moos, A. Jess, Direct detection of coking and regeneration of single particles and fixed bed reactors by electrical sensors, Applied Catalysis A: General 382, 254262 (2010); doi: 10.1016/j.apcata.2010.05.001

[5] N. Müller, A. Jess, R. Moos, Direct detection of coke deposits on fixed bed catalysts by electrical sensors, Sensors and Actuators B: Chemical, 144, 437-442 (2010); doi: 10.1016/j.snb.2009.03.008

[6] T. Oehmichen, Dissertation, Aachen, Shaker Verlag (2011); ISBN: 978-3-8322-9821-0
[7] http://www.chemie.de/lexikon/Nickel.html; 19. Dez. 2011

[8] http://www.chemie.de/lexikon/Nickel(II)sulfid.html; 19. Dez. 2011 